CORRELATION OF IN VIVO DELAYED-TYPE HYPERSENSITIVITY WITH IN VITRO LYMPHOCYTE TRANSFORMATION IN SARCOIDOSIS

O. P. SHARMA The cardinal immunological defect in sarcoidosis is depression of delayed-type hypersensitivity which is readily demonstrated by skin tests using tuberculin, Candida albicans, or the chemical dinitrochlorobenzene (DNCB). It is also known that in vitro culture of lymphocytes from sarcoidosis patients fail to undergo blastic transformation in response to phytohaemagglutinin (PHA). We have correlated the in vitro lymphocyte transformation to PHA with the in vivo response to DNCB in patients with sarcoidosis and in normal control subjects. There is a close correlation between in vitro and in vivo anergy, suggesting that the latter is due to a defect in cellular antibodies of the lymphocyte series.

A series of 21 patients in whom the diagnosis of sarcoidosis was supported by histological confirmation was compared with 17 control subjects who had no evidence of any disorder which might depress immune mechanisms. DNCB sensitization was performed initially with a $10 \%$ solution in acetone and tested four weeks later with a $0 \cdot 1 \%$ solution. The results of lymphocyte transformation were assessed by the percentage of blast cell transformation demonstrated by May-Grunwald-Giemsa stained smears and also by the uptake of tritiated thymidine (liquid scintillation counting). Fifteen of $21(71 \%)$ patients with sarcoidosis failed to develop DNCB sensitization which could be induced in $82 \%$ of the control subjects. In all but one control up to $90 \%$ lymphocytes showed blast formation and mitotic activity, whereas blast formation was under $70 \%$ in 19 of 21 $(90 \%)$ patients with sarcoidosis. There was a close correlation between the negative in vivo DNCB test and the sluggish in vitro lymphocyte response, both phenomena being noted in $71 \%$ of patients with sarcoidosis but in none of the controls. The ability to accept DNCB sensitization and have responsive lymphocytes was evident in three-quarters of controls and in only $10 \%$ of sarcoidosis patients.

\section{SERUM IMMUNOGLOBULIN (IgE) LEVELS IN ASTHMA}

S. G. O. JOHANSSON and H. BENNICH A new class of human immunoglobulins, $\operatorname{IgE}(\mathrm{E})$, has been found. Through the work of Ishizaka et al. and our group it has been shown that the skin-fixing (reaginic) antibodies usually belong to the IgE class.

The serum IgE concentration in healthy adults is very low, the mean value being $250 \mathrm{ng}$. $/ \mathrm{ml}$., which is about $1 / 50,000$ of the normal IgG concentration. Patients with bacterial, viral, and autoimmune diseases seem to have normal levels of IgE. This is in sharp contrast to atopic individuals, where mean IgE concentrations up to $2,800 \mathrm{ng}$. $/ \mathrm{ml}$. and single values of about $30,000 \mathrm{ng} . / \mathrm{ml}$. have been found.

In a preliminary study of asthmatic bronchitis it was found that patients with extrinsic asthma had a mean IgE level of $1,600 \mathrm{ng} . / \mathrm{ml}$., i.e., about six times the normal.
Patients with intrinsic asthma had normal levels. Further studies of children and adults with asthma and other $\frac{C}{\sigma}$ atopic diseases have shown a correlation between the $\overline{\bar{\omega}}$ severity of the disease and the IgE level. A rise in the $\frac{\mathscr{D}}{\mathbb{D}}$

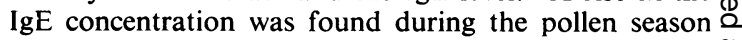
in patients with hay-fever and also after specific hyposensitization. The value of IgE determination in the $\overrightarrow{0}$ diagnosis of allergic diseases is discussed.

BRONCHIAL LAVAGE IN THE TREATMENT OF ASTHMA

E. SHERWOOD JONES In severe asthma airways obstruction is due to bronchospasm and bronchial plugging. if These cause respiratory failure and seriously interfere $\overrightarrow{0}$ with the filling and emptying of the heart, which may $\frac{1}{\sigma}$ arrest. Respiratory failure usually responds to con- 음 trolled oxygen, prednisolone, and fluid therapy. When this treatment fails and death is imminent, then controlled $c$ intermittent positive pressure ventilation (I.P.P.V.) is usually successful. I.P.P.V. is greatly facilitated by $\vec{c}$ bronchial lavage, which removes many of the mucous plugs. Experience with 50 patients has shown that bronchial lavage is simple and safe.

I.P.P.V. quickly relieves the mechanical stresses on the circulation and fully oxygenates the arterial blood; ventilation improves slowly over some hours.

MEDIASTINOSCOPY IN THE MANAGEMENT OF PATIENTS WITH
BRONCHIAL CARCINOMA

STURE LARSSON In patients with bronchial carcinoma and other forms of malignant tumour, the judgement as to prognosis, the planning of treatment, and the evaluation of results require a careful assessment of the extent $x$ of disease. A precise and complete description of the primary tumour as well as the regional lymph nodes and the distant metastases is necessary. In most patients a 0 radiologically detectable pulmonary opacity caused by the primary tumour is present at the date of diagnosis. At that time, however, many cases have already metastasized to regional lymph nodes and/or distant organs. Thus, detection of mediastinal lymph node metastases is of the utmost importance.

Mediastinoscopy has been shown to be a very valuable method for examination of the mediastinum. In our series of bronchial carcinoma mediastinoscopy was used $\omega$ routinely. These cases have been analysed and a prognosis is presented on the basis of these results Mediastinal lymph node metastases are usually a bad $\stackrel{0}{\varnothing}$ prognostic sign, but our investigation has shown that surgical treatment may be successful in certain cases with such metastases. A small group of patients with $-\overline{0}$ proved mediastinal lymph node metastases and perinodal $\stackrel{\overrightarrow{\mathbb{C}}}{\mathbb{T}}$ growth have received preoperative irradiation. The $\frac{\rho}{1}$ preliminary results are not encouraging. On the other hand, the results after resection are surprisingly favourable in some groups and pre-operative selection of these groups seems to be possible. 